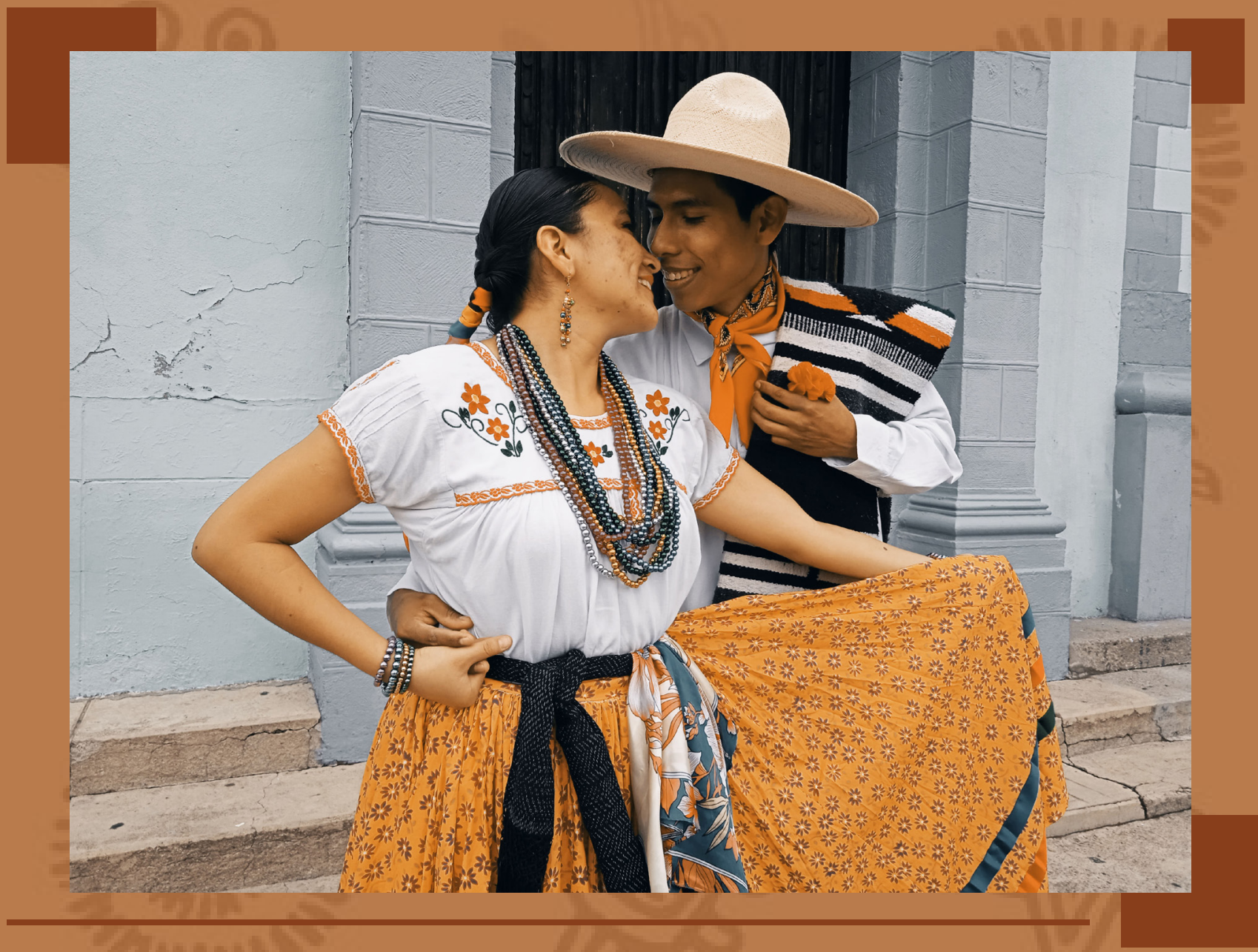

\title{
El jarabe Mixteco, ícono folclórico del Estado de Oaxaca
}




\section{El jarabe Mixteco, ícono folclórico del estado de Oaxaca}

\section{The jarabe Mixteco, folkloric icon of Oaxaca state}

Dulce María Clemente Guerrero

Universidad Tecnológica de la Mixteca

ID Orcid https://orcid.org/0000-0002-5740-6103

dulce@mixteco.utm.mx

\author{
Armando Rosas González \\ Universidad Tecnológica de la Mixteca \\ ID Orcid https://orcid.org/0000-0003-3034-2477 \\ arosas@mixteco.utm.mx
}

Recibido: 21-01-2021

Aceptado: 21-05-2021

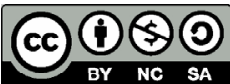

Copyright $\odot 2020$ UNAN-Managua Todos los Derechos Reservados.

\section{Resumen}

El "Jarabe Mixteco" es un compendio de sones y bailes alegres que se ha posicionado como la danza representativa de la región Mixteca debido a la expectación y aceptación que ha causado en el público nacional e internacional. Los siete sones que lo componen provienen del folclore popular de las celebraciones comunitarias regionales anteriores al siglo XX, sus coreografías dancísticas hacen referencia a representaciones de animales que son parte de la vida cotidiana de los pueblos mixtecos y también simbolizan el proceso de enamoramiento entre parejas (hombre y mujer). La indumentaria utilizada en su presentación posee formas, colores, materiales y simbología que expresan la identidad autóctona. El Jarabe Mixteco ha desempeñado el papel de embajador de la cultura mixteca y del estado de Oaxaca en todos los lugares en donde se ha presentado, ha tenido proyección mundial a través de su ejecución en el festival dancístico de la "Guelaguetza". El Jarabe Mixteco se ha convertido en un ícono del folclore mixteco ya que se vincula con la historia, las actividades y prácticas de la población; por ello, los ciudadanos, autoridades gubernamentales, organizaciones culturales y académicas se han encargado de investigar, compilar, presentar, preservar y difundir su legado cultural.

Palabras claves: Fotografía, danza, mixteco, folclore, México

\section{Abstract}

The Jarabe Mixteco is a compendium of sounds and joyful dances that has positioned itself as the representative dance of the Mixtec region due to the expectation and acceptance that it has caused in the national and international public. The seven sounds that compose it come from the popular folklore of regional community celebrations prior to the 20th century, the dance choreographies refer to representations of animals that are part of the daily life of mixtec peoples and also symbolize the process of falling in love between couples (man and woman). The clothing used in the presentation of this dance has shapes, colors, materials and symbols that express the native identity. The Jarabe Mixteco has played the role of ambassador of mixtec culture and the state of Oaxaca in all the places where it has been presented, this dance has had worldwide projection through its presentation at the "Guelaguetza" dance festival. The Jarabe Mixteco has become an icon of mixtec folklore as it is linked to the history, activities and practices of the population; for this reason, citizens, government authorities, cultural and academic organizations have been in charge of investigating, compiling, presenting, preserving and disseminating the cultural legacy of this dance.

Keywords: Photography, dance, mixtec, folklore, Mexico 


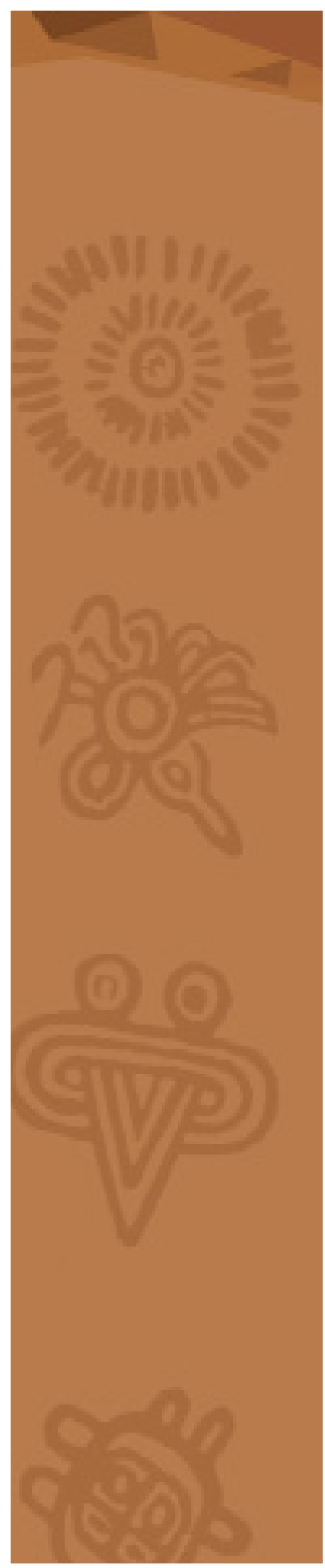

\section{Introducción}

A inicios del siglo XX surgió en México el "Jarabe Mixteco", compendio de sones y bailes alegres que se ha posicionado como la danza representativa de la región Mixteca debido a la expectación y aceptación que ha causado en el público nacional e internacional. Los registros históricos señalan que a partir del año 1922 se comenzó a presentar esta danza, se atribuye la recopilación de las coreografías dancísticas al maestro Cipriano Villa Hernández y de los arreglos musicales al maestro Antonio Martínez Corro. La mayoría de los sones que componen al Jarabe Mixteco hacen referencia a representaciones de animales que son parte de la vida cotidiana de los pueblos mixtecos y también simboliza el proceso de enamoramiento entre parejas (hombre y mujer). El Jarabe Mixteco ha tenido difusión y proyección mundial principalmente a través de su ejecución en la "Guelaguetza", el cual es el mayor festival dancístico del estado de Oaxaca (Durán, 2011; Herrera, 2019).

La indumentaria utilizada en el Jarabe Mixteco proviene de distintas localidades de la región; posee formas, colores, materiales y simbología que expresan la identidad autóctona: Las mujeres danzantes usan una blusa de manta con escote redondo que tiene elementos bordados en el cuello y en las mangas con hilos de distintos colores, en la parte frontal de esta prenda se coloca un pequeño peto; también las féminas visten una falda de tela de algodón de popelina con estampados florales, con corte de "holán al aire" y que tiene en el flanco inferior tres listones de diferentes colores que simbolizan a la mixteca oaxaqueña, poblana y guerrerense. Debajo de la falda generalmente las damas llevan un fondo de tela de manta, la falda se ciñe ocupando un rebozo negro, del cual cuelgan en el costado izquierdo siete listones multicolores que representan a cada una de las regiones geográficas del estado de Oaxaca. En el cuello, se colocan collares de "papelillo" multicolores y una mascada para secarse el sudor al danzar. Además, las mujeres calzan huaraches de correa blanca, su peinado se compone de un par de trenzas adornadas con cuatro listones y se colocan un clavel rojo en uno de los costados de la cabeza. Por su parte, el vestuario de los danzantes masculinos es más austero, ya que usan un pantalón y camisa de tela de manta, sobre uno de sus hombros cargan un gabán negro de lana, en el cuello y en la cintura se ciñen paliacates de color rojo, calzan huaraches de correo blanca y sobre su cabeza colocan un sombrero de palma.

Los siete sones musicales y la coreografía dancística que componen al Jarabe Mixteco provienen del folclore popular de las celebraciones comunitarias regionales anteriores al siglo XX; la ejecución de la danza se realiza por una pareja (hombre y mujer) acorde al siguiente orden: Los danzantes ingresa al escenario, caminando a paso lento, tomados de la mano uno a lado del otro, mientras una "banda de viento" interpreta la "Canción Mixteca", pieza musical que es considerada con el himno de facto de la región y que ha sido aclamada mundialmente. Posteriormente, los bailarines se ubican en el centro del escenario e inician la coreografía con el paso del "Giro Inicial" para quedar separados sobre una línea horizontal. A continuación, los danzantes ejecutan el son “El Macho", estos van cruzándose uno frente al otro a medida que realizan pasos rectos y cruzados, así se rememora la actividad de los antiguos arrieros de la región Mixteca, quienes transportaban diversos productos ocupando bestias de carga. Posteriormente, se presenta el son "El Chandé", en donde se imita el acto de persecución de un zorro (mujer) hacia un conejo (hombre), ambos bailarines realizan un taconeo rítmico. 


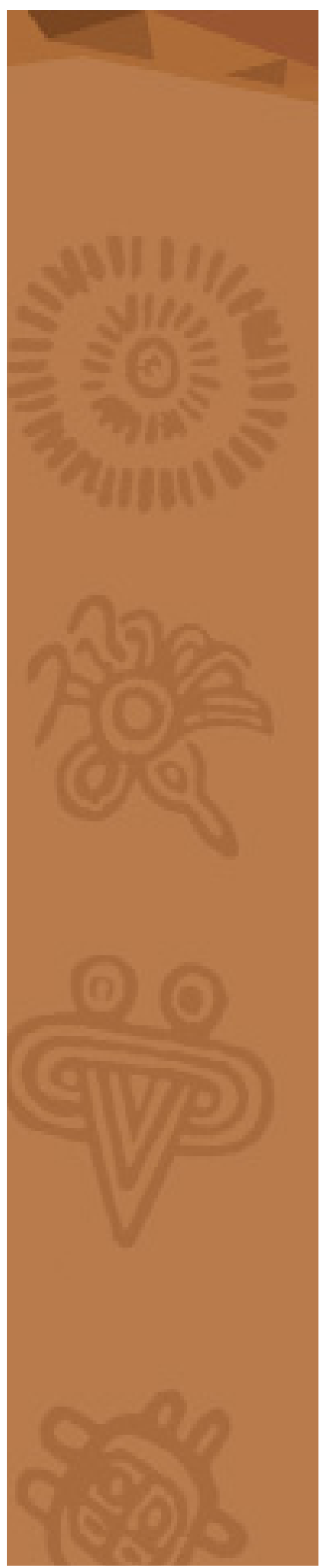

Después, se ejecuta el son "El Palomo", en donde la mujer coloca en su boca el clavel rojo que inicialmente tenía en la cabeza y es perseguida por el hombre hasta que éste logra quitárselo, se representa el acto de cortejo que realizan las aves. Luego, se presenta el son "El Oaxacado", en donde la mujer realiza movimientos llamativos con su falda, ambos bailarines hacen movimientos de ataque y defensa simulando la disputa entre un zopilote y un coyote por su presa. A continuación, se ejecuta el son "El Toro", en donde la mujer realiza una faena taurina al hombre usando la mascada colocada en su cuello. Finalmente, se ejecuta el son "Jarabe Final", ambos danzantes giran sobre su propio eje alternando saltos en un pie, hasta que la mujer da una vuelta final y cae en los brazos del hombre. La pareja de bailarines se despide del público ejecutando la misma coreografía hecha durante su entrada al escenario, mientras se vuelve a corear la Canción Mixteca (Casa de Cultura "Mtro. Antonio Martínez Corro", 2017; Castellanos et al., 2020).

A lo largo de más de noventa años, el Jarabe Mixteco ha desempeñado el papel de embajador de la cultura mixteca y del estado de Oaxaca en todos los lugares en donde se ha presentado. Los ciudadanos, autoridades gubernamentales, organizaciones culturales y académicas se han encargado de investigar, compilar, presentar, preservar y difundir su legado cultural. Esta danza causa júbilo en todos los sitios en los que se presenta, es una expresión cultural que se ha convertido en un ícono del folclore mixteco ya que se vincula con la historia, las actividades y prácticas de la población.

\section{Bibliografía}

- Casa de Cultura "Mtro. Antonio Martínez Corro", Jarabe Mixteco. Monografía, México.

- Castellanos, I., Ortíz, R., Bautista, L., Durán, J., 2020. El Jarabe Mixteco. Memorias y Documentos. Universidad Tecnológica de la Mixteca, México.

- Durán, M., 2011. Identidad y Cultura: la Alianza Cultural de Oaxaqueños por la Guelaguetza A. C. Tesis de grado, Centro de Investigaciones y Estudios Superiores de Antropología Socia Uso del robot humanoide NAO como herramienta de apoyo para la enseñanza del Jarabe Mixteco en niños de tercer grado de primaria. Tesis de grado, Universidad Tecnológica de la Mixteca, México. 


\section{Dulce María Clemente Guerrero}

Licenciada en Diseño Industrial por la Universidad Autónoma Metropolitana (2012), tiene un compromiso ético con la capacidad de entender y utilizar los códigos históricos, culturales y sociales. Maestra en Tecnología Avanzada de Manufactura por la Universidad Tecnológica de la Mixteca (2014). Es fotógrafa aficionada. Actualmente es profesora-investigadora de la Universidad Tecnológica de la Mixteca en el Instituto de Diseño y miembro del Cuerpo Académico UTMIX-CA-23 "Integración Multidisciplinaria del Diseño en México". Sus investigaciones se enfocan en el rescate de técnicas artesanales y el aprovechamiento de fibras naturales del estado de Oaxaca, México; para el diseño de objetos integrando el Ecodiseño.

\section{Armando Rosas González}

Licenciado en Ingeniería en Diseño por la Universidad Tecnológica de la Mixteca (2010). Maestro en Tecnología Avanzada de Manufactura por la Universidad Tecnológica de la Mixteca (2014). Es profesor-investigador del Instituto de Diseño de la Universidad Tecnológica de la Mixteca, miembro del Cuerpo Académico UTMIX-CA-23 "Integración Multidisciplinaria del Diseño en México". Es fotógrafo aficionado. Ha realizado investigaciones referentes al análisis y optimización de procesos, al desarrollo de nuevos productos y materiales, al análisis de la etnografía y su relación con el desarrollo de nuevos productos.

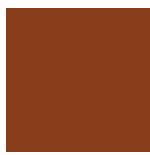




\section{Raíces}

Revista Nicaragüense de Antropología Año 5 No.9 | 2021 Enero - Junio

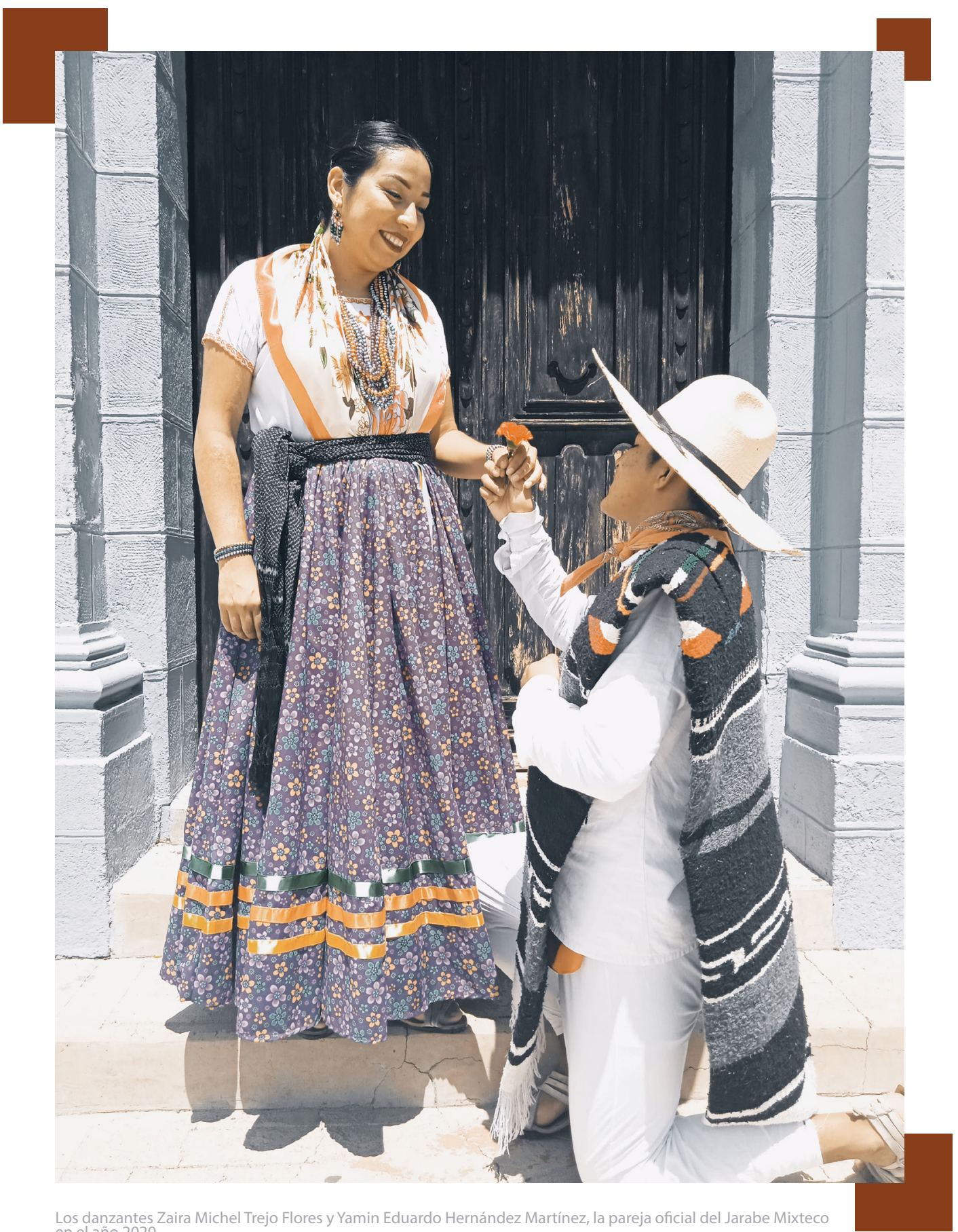



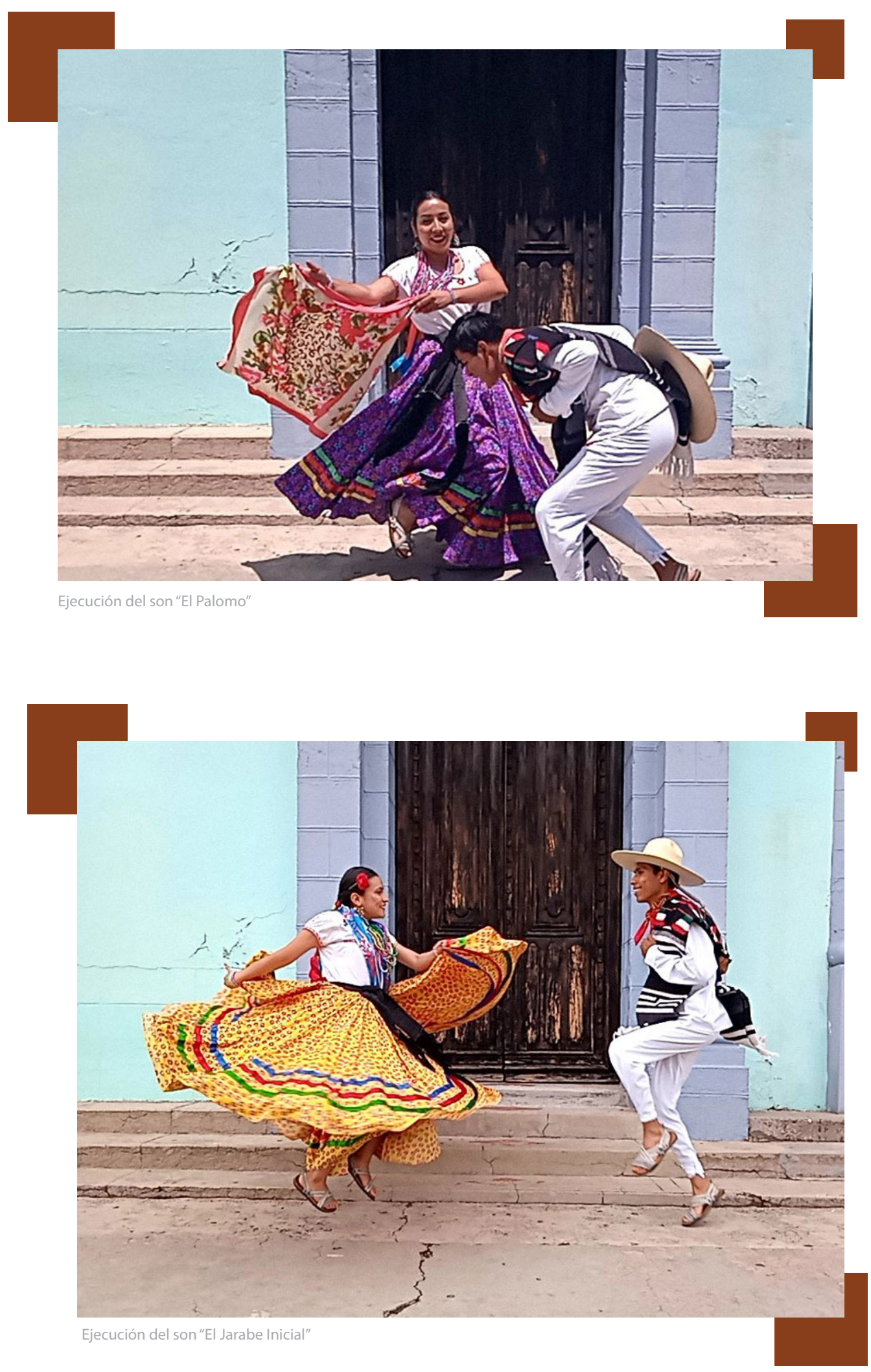


\section{Raíces}

Revista Nicaragüense de Antropología Año 5 No.9 | 2021 Enero - Junio
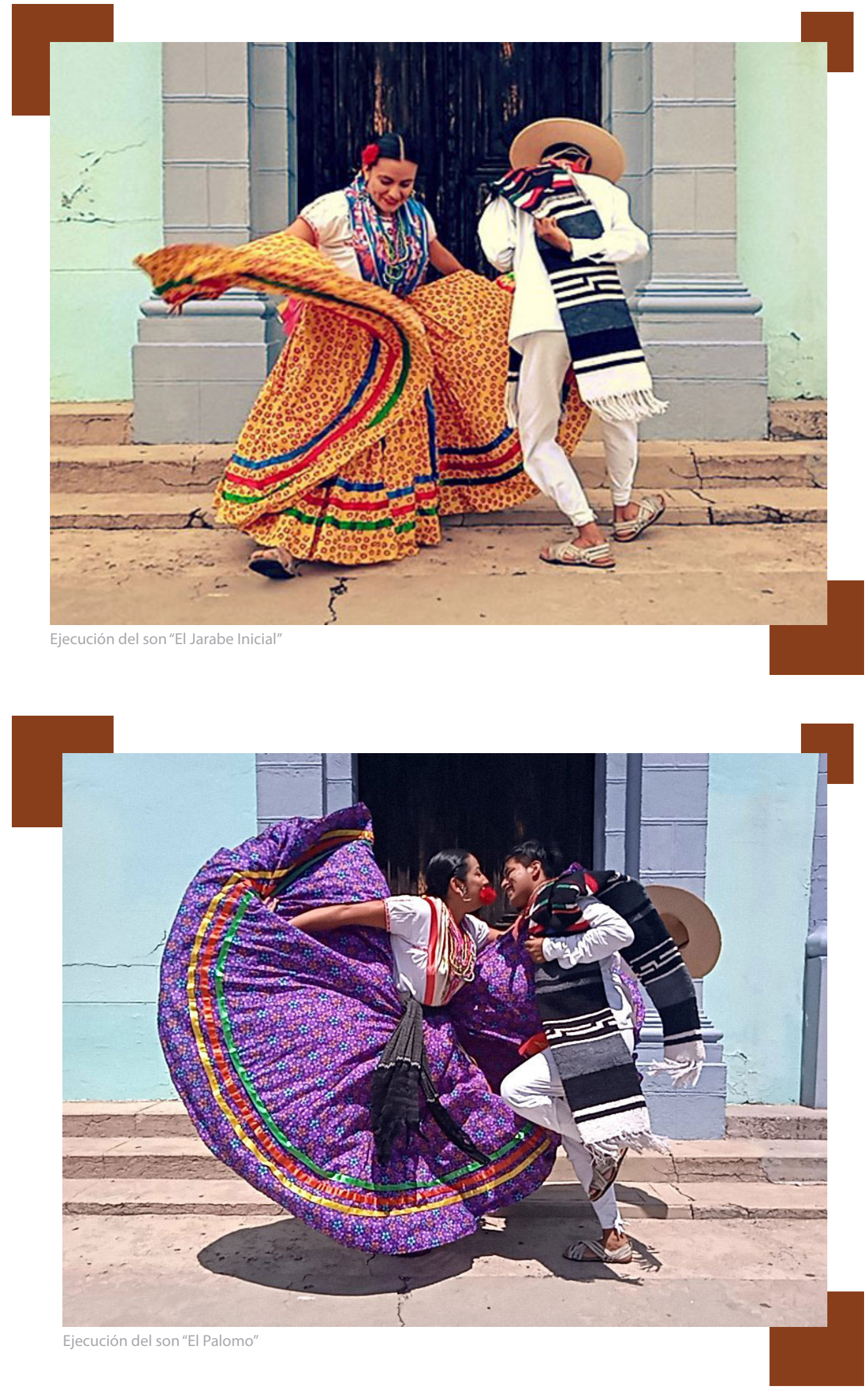